\title{
NONINVOLUTORIAL CREMONA TRANSFORMATIONS IN $[n]$
}

\section{EDWIN J. PURCELL}

There are, in the literature, many examples of noninvolutorial Cremona transformations in the plane, in ordinary space, and in higher space of definite dimension. But comparatively few such transformations are known for space of $n$ dimensions, where the $n$ is general.

This paper presents an extensive series of noninvolutorial Cremona transformations between two $n$-dimensional projective spaces ( $n$ any positive integer), developed by a new method, and classifies them. Very many known transformations may be obtained by assigning particular values to our general coefficients and by composition.

1. Algebraic introduction. Let

$$
\sum r_{l}=n \quad(l=1,2, \cdots, m)
$$

be an ordered partition of the positive integer $n$ into positive integral summands $r_{1}, r_{2}, \cdots, r_{m}$.

With $S_{0}=0$, let

$$
S_{l}=S_{l-1}+r_{l} \quad(l=1,2, \cdots, m) .
$$

Corresponding to the partition (1.1) of $n$ we separate the numbers $1,2, \cdots, n+1$ into $m$ sets of consecutive integers, the $l$ th set which corresponds to $r_{l}$ in (1.1) being

$$
S_{l-1}+1, S_{l-1}+2, S_{l-1}+3, \cdots, S_{l-1}+r_{l}+1 .
$$

By (1.2), $S_{l-1}+1=S_{l-2}+r_{l-1}+1$. Thus for $l>1$ the first integer of the $l$ th set is the last integer of the preceding set, and for $l<m$ the last integer of the $l$ th set is the first of the succeeding set. That is, the $m$ sets of consecutive integers (1.3) overlap.

Let the variable coordinates $\bar{x}_{1}, \bar{x}_{2}, \cdots, \bar{x}_{n+1}$ of a point $\bar{P}$ in a projective space $[\bar{n}]$ also be separated into sets corresponding to the sets (1.3) of their subscripts. If the $l$ th set $(l=1,2, \cdots, m)$ of $r_{l}+1$ variables

$$
\bar{x}_{S_{l-1}+1}, \bar{x}_{S_{l-1+2}}, \cdots, \bar{x}_{S_{l-1}+r_{l+1}}
$$

Received by the editors January 12, 1951 and, in revised form, November 17. 1951. 
satisfies the system of $r_{l}$ linear homogeneous equations (1.5) $\quad \sum_{j} a_{i j}^{(l)} \bar{x}_{S_{l-1}+j}=0 \quad\left(i=1,2, \cdots, r_{l} ; j=1,2, \cdots, r_{l}+1\right)$, we have the familiar solution

$$
\bar{x}_{S_{l-1}+j}=\rho_{l} A_{j}^{(l)},
$$

where $A_{j}^{(l)}$ is $(-1)^{j+1}$ times the determinant of order $r_{l}$ obtained by dropping the $j$ th column from the $\left(r_{l}, r_{l}+1\right)$ matrix $\left\|a_{i j}^{(l)}\right\|$. For our application to Cremona transformations it is necessary that

$$
A_{j}^{(l)} \neq 0 \quad\left(j=1,2, \cdots, r_{l}+1\right) .
$$

Now let each of the $m$ sets of variable coordinates (1.4) satisfy equations similar to (1.5), restricted as in (1.7) and with solutions (1.6). Then the solutions (1.6) yield a single solution $\bar{x}_{1}: \bar{x}_{2}: \cdots$ $: \bar{x}_{n+1}$. For, the overlapping variable in each of the $m-1$ pairs of consecutive systems yields a linear homogeneous equation

$$
\rho_{l} A_{r_{l+1}}^{(l)}=\rho_{l+1} A_{1}^{(l+1)} \quad(l=1,2, \cdots, m-1)
$$

between two consecutive factors of proportionality. The solution of these equations is easily verified to be

$$
\begin{aligned}
& \rho_{1}=\rho A_{1}^{(2)} A_{1}^{(3)} \cdots A_{1}^{(m)}, \\
& \rho_{l}=\rho A_{r_{1}+1}^{(1)} A_{r_{2}+1}^{(2)} \cdots A_{r_{l}+1+1}^{(l-1)} A_{1}^{(l+1)} A_{1}^{(l+2)} \cdots A_{1}^{(m)} \\
& \rho_{m}=\rho A_{r_{1}+1}^{(1)} A_{r_{2}+1}^{(2)} \cdots A_{r_{m-1}+1}^{(m-1)} .
\end{aligned}
$$

Thus the point $\bar{P}$ in $[\bar{n}]$ is completely determined by (1.6) and (1.9) in terms of the coefficients $a_{i j}^{(l)}$ of the $m$ sets of equations (1.5).

2. Cremona transformations. In the $m$ sets of equations (1.5) we define

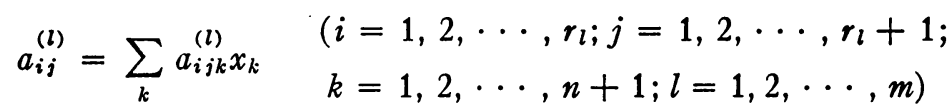

where $a_{i j t}^{(1)}$ are constants and, for $l>1, a_{i j k}^{(l)}$ are homogeneous polynomials of degree $\sigma_{l, t, i}$ in the $t$ th set of $\bar{x}^{\prime} \mathrm{s}(t=1,2, \cdots, l-1)$; the $x_{k}$ are the variable coordinates of a generic point $P$ in a projective space $[n]$.

In constructing our direct Cremona transformation, which sends $P$ into a point $\bar{P}$ of $[\bar{n}]$ ( $n=\bar{n}$ being any positive integer), we specify 
that the first set $(l=1)$ of equations, (1.5) with (2.1), be solved first and that the solution

$$
\bar{x}_{j}=\rho_{1} A_{j}^{(1)} \quad\left(j=1,2, \cdots, r_{1}+1\right)
$$

be substituted in the coefficients $a_{1 j k}^{(2)}\left(i=1,2, \cdots, r_{2} ; j=1,2, \cdots, r_{2}\right.$ $+1 ; k=1,2, \cdots, n+1)$ of the second set $(l=2)$ of equations.

Designate by $b_{i j k}^{(2)}$ the result of substituting the solution (2.2) in the $a_{i j k}^{(2)}$ and at the same time dropping the common factor $\rho_{1}^{\sigma, 1, i}$. Then form the equations

$$
\sum_{j} b_{i j}^{(2)} \bar{x}_{r_{1}+j}=0 \quad\left(i=1,2, \cdots, r_{2} ; j=1,2, \cdots, r_{2}+1\right)
$$

in which $b_{i j}^{(2)}=\sum_{k} b_{i j k}^{(2)} x_{k}(k=1,2, \cdots, n+1)$.

As in (1.5) and (1.6) the solution to (2.3) is

$$
\bar{x}_{r_{1}+j}=\rho_{2} B_{j}^{(2)},
$$

where $B_{j}^{(2)}$ is $(-1)^{j+1}$ times the determinant of order $r_{2}$ obtained by omitting the $j$ th column from the $\left(r_{2}, r_{2}+1\right)$ matrix $\left\|b_{i j}^{(2)}\right\|$.

Similarly, consider

$$
\sum_{j} b_{i j}^{(3)} \bar{x}_{S_{2}+j}=0 \quad\left(i=1,2, \cdots, r_{3} ; j=1,2, \cdots, r_{3}+1\right)
$$

in which $b_{i j}^{(3)}=\sum_{k} b_{i j k}^{(3)} x_{k}(k=1,2, \cdots, n+1)$ and the $b_{i j k}^{(3)}$ are obtained by substituting the solutions (2.2) and (2.4) in $a_{i j \mathbf{k}}^{(3)}$ and dropping the common factors $\rho_{1}^{\sigma_{3,1, i}}$ and $\rho_{2}^{\sigma_{3,2, i}}$.

Again as in (1.5) and (1.6) the solution to (2.5) is

$$
\bar{x}_{S_{2}+i}=\rho_{3} B_{i}^{(3)}
$$

where $B_{j}^{(3)}$ is $(-1)^{i+1}$ times the determinant of order $r_{3}$ formed by suppressing the $j$ th column in the $\left(r_{3}, r_{3}+1\right)$ matrix $\left\|b_{i j}^{(3)}\right\|$.

In general, we have

$$
\begin{array}{r}
\sum_{j} b_{i j}^{(l)} \bar{x}_{S_{l-1}+j}=0 \quad\left(i=1,2, \cdots, r_{l} ; j=1,2, \cdots, r_{l}+1 ;\right. \\
l=2,3, \cdots, m)
\end{array}
$$

wherein $b_{k j}^{(l)}=\sum_{k} b_{j k k}^{(l)} x_{k}(k=1,2, \cdots, n+1)$ and the $b_{j k \mathrm{k}}^{(l)}$ are the result of substituting the solutions (2.2), (2.4), (2.6), etc., in the $a_{i j k}^{(l)}$ while dropping the common factors $\rho_{h}^{\sigma_{l, h, i}}(h=1,2, \cdots, l-1)$. For uniformity of notation it is convenient to rewrite the $A_{j}^{(1)}, a_{i j}^{(1)}$, and $a_{i j \mathrm{k}}^{(1)}$ of $(2.2)$ as $B_{j}^{(1)}, b_{i j}^{(1)}$, and $b_{i j k}^{(1)}$, respectively. 
Thus the coefficients $b_{i j k}^{(l)}$ are polynomials homogeneous and of degree $\sigma_{l, 1, i}$ in $B_{1}^{(1)}, \ldots, B_{r_{1}+1}^{(1)}$, homogeneous and of degree $\sigma_{l, 2, i}$ in $B_{1}^{(2)}, \cdots, B_{r_{2}+1}^{(2)}$, etc., and finally of degree $\sigma_{l, l-1, i}$ in $B_{1}^{(2-1)}, \cdots$, $B_{r_{L 1}+1}^{(l-1)}$. The solution to $(2.7)$ is

$$
\bar{x}_{s_{l-1+j}}=\rho_{l} B_{j}^{(l)}
$$

where $B_{j}^{(l)}$ is $(-1)^{j+1}$ times the determinant of order $r_{l}$ obtained by dropping the $j$ th column from the $\left(r_{l}, r_{l}+1\right)$ matrix

$$
\left\|b_{i j}^{(l)}\right\| \text {. }
$$

The equations of the direct transformation, which follow from (2.8) and equations similar to (1.9), are

$$
\begin{array}{r}
\bar{x}_{j}=\rho B_{j}^{(1)} B_{1}^{(2)} B_{1}^{(3)} \cdots B_{1}^{(m)} \quad\left(j=1,2, \cdots, r_{1}+1\right), \\
\bar{x}_{S_{l-1}+j}=\rho B_{r_{1}+1}^{(1)} B_{r_{2}+1}^{(2)} \cdots B_{r_{l-1}+1}^{(l-1)} B_{j}^{(l)} B_{1}^{(l+1)} B_{1}^{(l+2)} \cdots B_{1}^{(m)} \\
\left(j=1,2, \cdots, r_{l}+1 ; l=2,3, \cdots, m-1\right), \\
\bar{x}_{S_{m-1}+j}=\rho B_{r_{1}+1}^{(1)} B_{r_{2}+1}^{(2)} \cdots B_{r_{m-1}+1}^{(m-1)} B_{j}^{(m)}\left(j=1,2, \cdots, r_{m}+1\right) .
\end{array}
$$

The transformations are classified by means of the type symbol

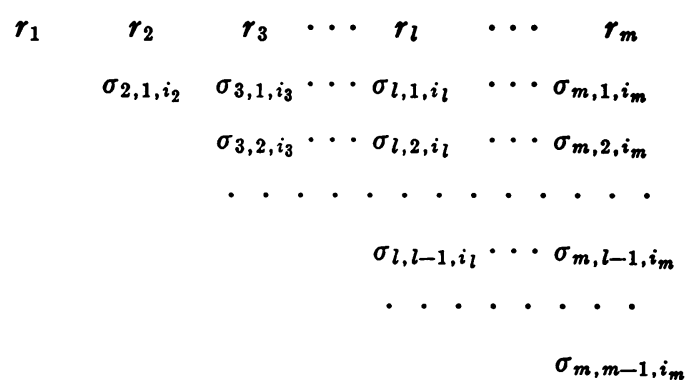

where $\sigma_{l, t, i_{l}}=\sigma_{l, t, 1}, \sigma_{l, t, 2}, \cdots, \sigma_{l, t, r_{l}}$ and in which the $\sigma_{c, d, i}$ s are any positive integers or zero, save only that when $d=c-1$, not all the $\sigma_{c, c-1, i}$ 's can be zero for any particular value of $c$. For each given ordered partition of $n$ we have one type of transformation. In $[n]$ there are $2^{n-1}$ types of our Cremona transformations.

Let $d_{l}$ be the degree in $x$ of the determinants $B_{1}^{(l)}, B_{2}^{(l)}, \ldots, B_{r_{l}+1}^{(l)}$. We have, then, the recursion formula

$$
\begin{array}{r}
d_{l}=r_{l}+d_{1} \sum_{i} \sigma_{l, 1, i}+d_{2} \sum_{i} \sigma_{l, 2, i}+\cdots+d_{l-1} \sum_{i} \sigma_{l, l-1, i} \\
\left(i=1,2, \cdots, r_{l}\right) .
\end{array}
$$


From (2.10) it is clear that the order of the direct transformation, from $[n]$ to $[\bar{n}]$, is

$$
Q=d_{1}+d_{2}+\cdots+d_{l}+\cdots+d_{m} .
$$

A little consideration of early cases, $d_{1}, d_{2}, d_{3}, d_{4}$, and their sums yields the following result:

$$
Q=\sum_{k}\left\{r_{j_{k}} \prod_{l}\left(\sum_{i} \sigma_{j_{l-1}, j_{l}, i}\right)\right\}
$$

$\left(k=1,2, \cdots, m ; l=2,3, \cdots, k ; i=1,2, \cdots, r_{j_{l-1}} ; j_{1} \leqq m ; j_{1}>j_{2}\right.$ $\left.>\cdots>j_{k} ; \sum_{1}=r_{1}+r_{2}+\cdots+r_{m}\right)$. This notation indicates that for given $k, j_{1}, j_{2}, \cdots, j_{k}$ take on all sets of $k$ positive integral values subject to the above conditions. For example, when $k=3$ and $m=4$, $j_{1}, j_{2}, j_{3}$ take on the sets of values $4,3,2 ; 4,3,1 ; 4,2,1 ; 3,2,1$.

We now seek the equations of the inverse transformation from $[\bar{n}]$ to $[n]$. The $m$ sets of $r_{l}$ equations, (1.5) with (2.1), for $l=1,2, \cdots, m$, are linear in $x_{1}, \cdots, x_{n+1}$ and their number is $\sum r_{l}=n$. They may be written in the form

$$
\begin{gathered}
m_{1,1} x_{1}+\cdots+m_{1, n+1} x_{n+1}=0, \\
\cdot \cdot \cdot \cdot \cdot \cdot \cdot \cdot \cdot \cdot \cdot \cdot, \\
m_{n, 1} x_{1}+\cdots+m_{n, n+1} x_{n+1}=0 .
\end{gathered}
$$

Thus the equations of the inverse transformation are

$$
x_{a}=\tau M_{a} \quad(a=1,2, \cdots, n+1),
$$

where $M_{a}$ is $(-1)^{a+1}$ times the determinant of order $n$ formed from the $(n, n+1)$ matrix $\left\|m_{i, j}\right\|$ by omitting the $a$ th column, and $\tau$ is a factor of proportionality.

The order $\bar{Q}$ of this inverse transformation is the order of the determinants $M_{a}$. The first $r_{1}$ equations are linear in $\bar{x}_{1}, \cdots, \bar{x}_{r_{1}+1}$ and they contribute $r_{1}$ to the order $\bar{Q}$. The second set of $r_{2}$ equations are linear in $\bar{x}_{S_{1+1}}, \cdots, \bar{x}_{S_{1+r_{2}+1}}$ and of degree $\sigma_{2,1, i}$ in $\bar{x}_{1}, \cdots, \bar{x}_{r_{1}+1}$ $\left(i=1,2, \cdots, r_{2}\right)$ [cf. (2.1)]. Thus they contribute $r_{2}+\sum_{i} \sigma_{2,1, i}$ to $\bar{Q}$. Similarly the contribution of the third set of $r_{3}$ equations to $\bar{Q}$ is $r_{3}+\sum_{i} \sigma_{3,1, i}+\sum_{i} \sigma_{3,2, i}\left(i=1,2, \cdots, r_{3}\right)$. Proceeding onward we find that the order of the inverse transformation is

$$
\begin{aligned}
\bar{Q}=n+\sum_{i} \sigma_{l, 1, i}+\sum_{i} \sigma_{l, 2, i}+\cdots+\sum_{i} \sigma_{l, l-1, i} \\
\quad\left(i=1,2, \cdots, r_{l} ; l=2,3, \cdots, m\right) .
\end{aligned}
$$

A necessary condition that the transformation from $[\bar{n}]$ to $[n]$ 
exist is that $M_{a} \neq 0(a=1,2, \cdots, n+1)$. Should any $M_{a}$ vanish identically, the equations (2.16) would map the $[\bar{n}]$ of $\bar{P}$ onto the prime $x_{a}=0$ in $[n]$ and thus not be a Cremona transformation.

For this same reason the restrictions of (1.7) are necessary for $l=1,2, \cdots, m ; j=1,2, \cdots, r_{l}+1$. Otherwise the situation just mentioned would occur in the direct transformation from $[n]$ to $[\bar{n}]$ since any $B_{j}^{(l)}$ occurs in at least one of the $\bar{x}_{s_{l-1}+j}$.

3. The fundamental and principal systems. The $r_{1}+1$ simultaneous equations $B_{j}^{(1)}=0\left(j=1,2, \ldots, r_{1}+1\right)$ define a variety $B^{(1)}$. The $r_{2}+1$ equations $B_{j}^{(2)}=0\left(j=1,2, \cdots, r_{2}+1\right)$ determine a locus consisting of $B^{(1)}$ multiply and a residual variety $B^{(2)}$. Similarly the locus of the $r_{l}+1$ simultaneous equations $B_{j}^{(l)}=0 \quad\left(j=1,2, \cdots, r_{l}+1\right.$; $l=1,2, \cdots, m)$ consists of $B^{(t)}(t=1,2, \cdots, l-1)$ multiply and a residual variety $B^{(l)}$. The $B^{(l)}(l=1,2, \cdots, m)$ are fundamental varieties in $[n]$. They are all of dimension $n-2$.

To find the order of the fundamental variety $B^{(l)}$ we shall first consider any nonspecial $b_{i j}^{(l)}(l=1,2, \cdots, m)$ and designate its total order in $x_{1}, \cdots, x_{n+1}$ by $b^{(l, i)}$. Then $b^{(1, i)}=1$ and, for $l>1, b^{(l, i)}$ is given by the recurrence formula

$$
b^{(l, i)}=\sum_{v}\left\{\sigma_{l, v, i}\left(\sum_{h} b^{(v, h)}\right)\right\}+1
$$

$\left(v=1,2, \cdots, l-1 ; h=1,2, \cdots, r_{v} ; l=2,3, \cdots, m\right)$. Indicate the total multiplicity of $B^{(k)}$ on $b_{i j}^{(l)}$ by $b_{k}^{(i, 1)}(k=1,2, \cdots, l-1)$. For $l>2, b_{k}^{(l, k)}$ is shown by the recurrence formula

$$
b_{k}^{(l, i)}=\sigma_{l, k, i}+\sum_{w}\left\{\sigma_{l, w, i}\left(b_{k}^{(w, 1)}+\cdots+b_{k}^{\left(w, r_{w}\right)}\right)\right\}
$$

$(w=k+1, k+2, \cdots, l-1 ; k=1,2, \cdots, l-2)$ and $b_{l-1}^{(, i)}=\sigma_{l, l-1, i}$. If we designate the order of $B^{(k)}$ by $\beta_{k}$ and denote by $F\left(b^{(l, i)}\right)$ the expression

$$
\begin{aligned}
& \left\{\left(\sum_{i=1}^{r l} b^{(l, i)}\right)^{2}+\left(\sum_{i=3}^{r l} b^{(l, i)}\right)^{2}+\left(\sum_{i=5}^{r l} b^{(l, i)}\right)^{2}+\cdots\right\} \\
& \quad-\left\{\left(b^{(l, 1)}+\sum_{i=3}^{r l} b^{(l, i)}\right)\left(b^{(l, 2)}+\sum_{i=3}^{r l} b^{(l, i)}\right)\right. \\
& \quad+\left(b^{(l, 3)}+\sum_{i=5}^{r l} b^{(l, i)}\right)\left(b^{(l, 4)}+\sum_{i=5}^{r l} b^{(l, i)}\right) \\
& \left.\quad+\left(b^{(l, 5)}+\sum_{i=7}^{r l} b^{(l, i)}\right)\left(b^{(l, 6)}+\sum_{i=7}^{r l} b^{(l, i)}\right)+\cdots\right\},
\end{aligned}
$$


then the order of $B^{(l)}$ for $l>1$ is given by the recurrence formula

$$
F\left(b^{(l, i)}\right)-\sum_{k}\left\{\beta_{k} F\left(b_{k}^{(l, i)}\right)\right\}
$$

$(k=1,2, \cdots, l-1 ; l=2,3, \cdots, m)$, and the order of $B^{(1)}$ is $r_{1}\left(r_{1}+1\right) / 2$.

The multiplicity of $B^{(l)}$ on the homaloids in $[n]$ is

$$
1+\sum_{k}\left\{\left(\sum_{i=1}^{r_{j_{1}}} \sigma_{j_{1}, j_{2}, i}\right)\left(\sum_{i=1}^{r_{i_{2}}} \sigma_{j_{2}, j_{3}, i}\right) \cdots\left(\sum_{i=1}^{r_{j_{k}}} \sigma_{j_{k}, l, i}\right)\right\}
$$

$\left(k=1,2, \cdots, m-l ; k+l \leqq j_{1} \leqq m ; j_{1}>j_{2}>\cdots>j_{k}>l ; l=1,2, \cdots\right.$, $m-1)$. The multiplicity of $B^{(m)}$ on the homaloids in $[n]$ is 1 .

The principal variety in $[\bar{n}]$, image of the fundamental variety $B^{(l)}$ in $[n]$, is a primal of order $r_{l} \bar{Q}+\sum_{i}\left(\sigma_{l, 1, i}+\sigma_{l, 2, i}+\cdots+\sigma_{l, l-1, i}\right)$ $-1\left(i=1,2, \cdots, r_{l} ; l=1,2, \cdots, m\right)$.

Other fundamental varieties in $[n]$ arise from the overlapping determinants. The varieties $B_{r_{l}+1}^{(l)}=B_{1}^{(l+1)}=0(l=1,2, \cdots, m-1)$ are fundamental varieties in $[n]$. They are of dimension $n-2$ and are simple on the homaloids in $[n]$.

The image in $[\bar{n}]$ of the $F$-variety $B_{r_{l}+1}^{(l)}=B_{1}^{(l+1)}=0$ in $[n]$ is the $P$-prime $\bar{x}_{S_{l+1}}=0$.

Fundamental elements in $[n]$ whose images are themselves $F$-elements in $[\bar{n}]$ are $B_{r_{l+1}}^{(l)}=B_{1}^{(k)}=0(k=l+2, l+3, \cdots, m ; l=1,2, \cdots$, $m-2)$. Here, for each value of $l$ from 1 to $m-2, k$ takes on every integral value from $l+2$ to $m$, inclusive. They are of dimension $n-2$ and are simple on the homaloids in $[n]$.

The $P$-varieties in $[\bar{n}]$ that correspond to the $F$-varieties $B_{r_{b}+1}^{(l)}$ $=B_{1}^{(k)}=0$ in $[n]$ are the $F$-varieties in $[\bar{n}]$ whose equations are $\bar{x}_{S_{l+1}}=\bar{x}_{S_{l+2}}=\cdots=\bar{x}_{S_{k-1+1}}=0$.

As to the $F$-system in $[\bar{n}]$, the flat space of dimension $S_{l}-1$ whose equations are $\bar{x}_{S_{l+1}}=\bar{x}_{S_{l+2}}=\cdots=\bar{x}_{n+1}=0(l=1,2, \cdots, m-1)$ is fundamental. Its multiplicity on the homaloids in $[\bar{n}]$ is

$$
\sum_{j}\left\{r_{j}+\sum_{i}\left(\sigma_{j, l+1, i}+\sigma_{i, l+2, i}+\cdots+\sigma_{j, j-1, i}\right)\right\}
$$

$\left(l=1,2, \cdots, m-1 ; j=l+1, l+2, \cdots, m ; i=1,2, \cdots, r_{i}\right)$.

The $P$-primal in $[n]$ that corresponds to this $F$-flat in $[\bar{n}]$ is $B_{r_{l}+1}^{(l)}=0$.

Also the flat space whose equations are $\bar{x}_{1}=\bar{x}_{2}=\cdots=\bar{x}_{S_{l-1+1}}=0$ $(l=2,3, \cdots, m)$ is fundamental in $[\bar{n}]$. It is of dimension $n-S_{l-1}-1$. Its multiplicity on the homaloids in $[\bar{n}]$ is 


$$
\sum_{j}\left\{r_{i}+\sum_{i}\left(\sigma_{i, 1, i}+\sigma_{i, 2, i}+\cdots+\sigma_{j, j-1, i}\right)\right\}
$$

$\left(j=1,2, \cdots, m ; i=1,2, \cdots, r_{j} ; l=2,3, \cdots, m\right)$. Its $P$-primal in $[n]$ is $B_{1}^{(l)}=0$.

$F$-element in $[\bar{n}]$ whose image is an $F$-element of $[n]$ is the flat space of dimension $n-\left(r_{l+1}+r_{l+2}+\cdots+r_{k-1}+1\right)$ whose equations are $\bar{x}_{S_{l+1}}=\bar{x}_{S_{l+2}}=\cdots=\bar{x}_{S_{k-1}+1}=0(k=l+2, l+3, \cdots, m ; l=1,2$, $\cdots, m-2)$. It joins the $F$-elements $\bar{x}_{s_{l+1}}=\bar{x}_{s l+2}=\cdots=\bar{x}_{n+1}=0$ and $\bar{x}_{1}=\bar{x}_{2}=\cdots=\bar{x}_{S_{k-1}+1}=0$. Its being an $F$-element is a necessary consequence of the multiplicity of the two just-mentioned $F$-elements on the homaloids in $[\bar{n}]$.

The $P$-elements in $[n]$ corresponding to these $F$-elements in $[\bar{n}]$ are the $F$-elements $B_{r_{l}+1}^{(l)}=B_{1}^{(k)}=0$.

There is another $F$-element in $[\bar{n}]$ which will be indicated by $M$. It is a variety of dimension $n-2$ and is simple on the homaloids in $[\bar{n}] . M$ is the locus of points in $[\bar{n}]$, other than the $F$-elements of $[\bar{n}]$ already considered, that cause the $(n, n+1)$ matrix $\left\|m_{i j}\right\|$ to be of rank $n-1(i=1,2, \cdots, n ; j=1,2, \cdots, n+1)$.

The $P$-primal in $[n]$ that corresponds to $M$ is a manifold consisting of all lines that intersect $r_{l}$ times every $B^{(l)}(l=1,2, \cdots, m)$.

For $n$ sufficiently great there are points of $[\bar{n}]$ whose coordinates cause the rank of $\left\|m_{i j}\right\|$ to be less than $n-1$, say rank $R$. The points of $[\bar{n}]$ that cause $\left\|m_{i j}\right\|$ to be of rank $R(R<n-1)$ form a locus of dimension less than $n-2$. Such a locus is an $F$-element in $[\bar{n}]$ and is contained multiply in $M$.

4. Congruences. It will be noted that when $m>1$, the first $m-1$ sets of equations, (1.5) with (2.1), define a flat space congruence of order one in $[n] .{ }^{1}$ Through a generic point of $[n]$ one and only one $S_{r_{m}}$ of the congruence passes. The carriers of this congruence are the $B^{(l)}(l=1,2, \cdots, m-1)$.

In $[\bar{n}]$ the fundamental flat space $S_{r_{m}-1}$, whose equations are $\bar{x}_{1}=\bar{x}_{2}=\cdots=\bar{x}_{S_{m-1}+1}=0$, is to multiplicity

$$
n-r_{m}+\sum_{j}\left\{\sum_{i}\left(\sigma_{j, 1, i}+\sigma_{j, 2, i}+\cdots+\sigma_{i, j-1, i}\right)\right\}
$$

$\left(i=1,2, \cdots, r_{j} ; j=2,3, \cdots, m\right)$ on the homaloids. A generic point $\bar{P}$ of $[\bar{n}]$ determines with this $S_{r_{m}-1}$ a unique $S_{r_{m}}$ of the first order congruence of $S_{r_{m}}$ 's on the $S_{r_{m}-1}$. All nonspecial points on the $S_{r_{m}}$

1 E. J. Purcell, Flat space congruences of order one in [n], Trans. Amer. Math. Soc. vol. 54 (1943) pp. 57-69. 
determined by $\bar{P}$ have $\bar{x}_{1}, \bar{x}_{2}, \cdots, \bar{x}_{S_{m-1}+1}$ in constant ratio and therefore determine a unique $S_{r_{m}}$ of the congruence in $[n]$, and conversely. All nonspecial points on an $S_{r_{m}}$ of the congruence in either space go over into the points of a unique $S_{r_{m}}$ of the congruence in the other space. That is, when $m>1$, the Cremona transformations send the $S_{r_{m}}$ 's of a congruence of order one in either space into the $S_{r_{m}}$ 's of a congruence of order one in the other space.

Moreover, although the C.T. does not in general send the points of the $S_{n-r_{m}}$ in $[n]$ whose equations are $x_{n-r_{m}+2}=x_{n-r_{m}+3}=\cdots=x_{n+1}=0$ into the points of the $S_{n-r_{m}}$ in $[\bar{n}]$ whose equations are $\bar{x}_{n-r_{m}+2}$ $=x_{n-r_{m}+3}=\cdots=\bar{x}_{n+1}=0$, the congruences do relate the points of these two $S_{n-r_{m}}$ 's in a type

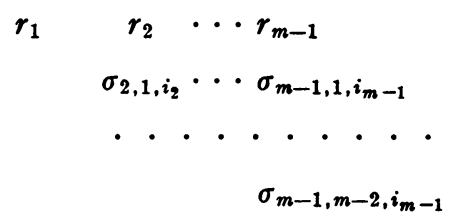

Cremona transformation whose direct equations may be obtained from the first $n-r_{m}+1$ equations of (2.10) by setting $x_{n-r_{m}+2}=x_{n-r_{m}+3}$ $=\cdots=x_{n+1}=0$, and whose inverse equations are found by substituting $\bar{x}_{n-r_{m}+2}=\bar{x}_{n-r_{m}+3}=\cdots=\bar{x}_{n+1}=0$ in the first $n-r_{m}+1$ equations of (2.16).

5. $n=1,2$, and 3. It is to be emphasized that the Cremona transformations just discussed are of great generality. Very many known C.T's appear as special cases whose equations may be obtained by assigning particular values to our general coefficients and parameters. Still others result from composition. However, the task of examining systematically all known space Cremona transformations in relation to our types has not been attempted.

When $n=1$, there is just one type and its type symbol (2.11) is 1 . It is the ordinary binary projectivity, the only transformation which should appear in $S_{1}$ in any sequence of Cremona transformations depending on $n$.

In the plane there are two types of Cremona transformations. Type symbol 2 gives the plane quadratic transformation from which, as Noether showed, all other plane C.T.'s can be compounded. Type

$$
\begin{array}{cc}
1 & 1 \\
& \sigma_{2,1,1}
\end{array}
$$

is the plane de Jonquieres' transformation whose order is $\sigma_{2,1,1}+2$, 
where $\sigma_{2,1,1}$ is any positive integer.

In three-dimensional space there are four types as indicated by the type symbols

$$
\begin{aligned}
& 3, \quad 2 \quad 1, \begin{array}{llllllll} 
& 2 & 2 & \text { and } & 1 & 1 & 1 .
\end{array} \\
& \sigma_{2,1,1} \quad \sigma_{2,1, i}(i=1,2) \quad \sigma_{2,1,1} \quad \sigma_{3,1,1} \\
& \sigma_{3,2,1}
\end{aligned}
$$

Type 3 is the familiar bilinear cubo-cubic space Cremona transformation whose $F$-system in either space is a sextic curve. The many variations of this transformation that arise when the $F$-sextic degenerates can all be obtained from our general type 3 by assigning particular values to the coefficients.

Even the quadro-quadric space C.T. is a special case of our type 3, obtained by setting $a_{14}^{(1)}=a_{24}^{(1)}=a_{31}^{(1)}=a_{32}^{(1)}=0$ in (1.5) and (2.1). It has a conic and a point not in the plane of the conic as $F$-elements in either space. The variations that arise when the $F$-conic degenerates are obtained by further specialization of the remaining parameters. A large number of known space Cremona transformations can be compounded from this $T_{2-2}$.

The $T_{2-3}$, also, can be obtained by specializing the arbitrary constants in our general type 3. It may also be compounded of $T_{2-2}$ 's.

Another useful specialization of our type 3 is the tetrahedral transformation, whose equations may be formed from (1.5) and (2.1) by letting $a_{11}^{(1)}, a_{12}^{(1)}, a_{22}^{(1)}, a_{23}^{(1)}, a_{33}^{(1)}$, and $a_{34}^{(1)}$ be $x_{1},-x_{2}, x_{2},-x_{3}, x_{3}$, and $-x_{4}$, respectively, while all the other $a_{i j}^{(1)}$ 's are zero. Equations (2.10) and (2.16) of the direct and inverse transformation become those given by Hudson. ${ }^{2}$ The order is 3 in both directions and the fundamental sextic curve of our general type 3 here degenerates into six lines, the edges of the tetrahedron of reference. All the so-called punctual transformations are, by definition, ${ }^{3}$ compounded of these tetrahedral transformations.

Before leaving this first type of C.T. in [3], it is to be noted that our type $n$ C.T. in $[n]$ has been studied by Godeaux ${ }^{4}$ from another viewpoint.

The second type of Cremona transformation in $S_{3}$ is type 301.

${ }^{2}$ H. Hudson, Cremona transformations, Cambridge University Press, 1927, p.

${ }^{3}$ Hudson, loc. cit., p. 318.

4 L. Godeaux, Sur une correspondance Crémonienne entre deux espaces d $n$ dimensions, Istituto Lombardo di Scienze e Lettere. Rendiconti (2) vol. 43 (1910) pp. 116119. 


$$
\begin{array}{cc}
2 & 1 \\
& \sigma_{2,1,1}
\end{array} .
$$

It is a series of transformations very similar to those of Tummarello. ${ }^{5}$ The order of the direct transformation is $2 \sigma_{2,1,1}+3$ and that of the inverse transformation is $\sigma_{2,1,1}+3$, where $\sigma_{2,1,1}$ is any positive integer. The homaloidal web in [3] consists of de Vries surfaces and the web in $[\overline{3}]$ is monoidal. $B^{(1)}$, a twisted cubic curve, is a $\left(\sigma_{2,1,1}+1\right)$-fold fundamental curve. Its principal surface in $[\overline{3}]$ is of order $2 \sigma_{2,1,1}+5$, on which (0001) is $\left(2 \sigma_{2,1,1}+3\right)$-fold point and line $\bar{x}_{3}=\bar{x}_{4}=0$ is double. $B^{(2)}$ is a simple $F$-curve of order $\sigma_{2,1,1}^{2}+4 \sigma_{2,1,1}+1$. Its $P$-surface in $[\overline{3}]$ is a cone of order $2 \sigma_{2,1,1}+2$, whose vertex is (0001) and whose base curve is $M . B^{(2)}$ intersects $B^{(1)}$ in $2 \sigma_{2,1,1}^{2}+6 \sigma_{2,1,1}$ points. $B_{3}^{(1)}$ $=B_{1}^{(2)}=0$ is a simple $F$-curve of order $\sigma_{2,1,1}+2$. This curve intersects $B^{(1)}$ in $2 \sigma_{2,1,1}+3$ points and intersects $B^{(2)}$ in $2 \sigma_{2,1,1}+2$ points. Its $P$-surface is the plane $\bar{x}_{3}=0$.

The homaloids in [3] are monoidal surfaces of order $\sigma_{2,1,1}+3$. On them $(0001)$ is an isolated $\left(\sigma_{2,1,1}+2\right)$-fold $F$-point, $\bar{x}_{3}=\bar{x}_{4}=0$ is a simple $F$-line, and $M$ is a simple $F$-curve. The order of $M$ is $\sigma_{2,1,1}^{2}$ $+4 \sigma_{2,1,1}+5$ and on it $(0001)$ is a $\left(\sigma_{2,1,1}^{2}+2 \sigma_{2,1,1}+3\right)$-fold point. Its $P$-surface in [3] is ruled and consists of all lines that are bisecants of $B^{(1)}$ and intersect $B^{(2)}$. This ruled surface has $B^{(1)}$ as $\left(2 \sigma_{2,1,1}+2\right)$-fold directrix curve and $B^{(2)}$ as simple directrix curve. Its order is $4 \sigma_{2,1,1}$ +4 . The $P$-surface of $\bar{x}_{3}=\bar{x}_{4}=0$ is the quadric $B_{3}^{(1)}=0$ in [3]. The $P$-surface of $(0001)$ is $B_{1}^{(2)}=0$, of order $2 \sigma_{2,1,1}+1$ and containing $B^{(1)}$ as $\sigma_{2,1,1}$-fold curve and $B^{(2)}$ as simple curve.

This transformation sends the rays of the line congruence of order one consisting of the bisecants of the twisted cubic $B^{(1)}$ into the rays of star on (0001) in [ $\overline{3}$ ], and conversely. Moreover, the coefficients of this transformation can be specialized so that the transformation becomes bi-monoidal. When all the $a_{i j}^{(1)}=0$ are planes through the same point 0 in [3], the congruence of bisecants of the twisted cubic $B^{(1)}$ becomes the star of lines on $0 .{ }^{6}$

The third type of C.T. in $S_{3}$ is

$$
12 .
$$

It sends the planes of the pencil on $B^{(1)}$ in [3] over into the planes

5 A. Tummarello, Una nuova trasformazione birazionale $(n, 2 n-3)$ dello spazio a tre dimensioni. Le superficie di de Vries, Catania Circ. M. Note e Mem. vol. 1, pp. 282288.

${ }^{6}$ Purcell, loc. cit., p. 67. 
of the pencil on $\bar{x}_{1}=\bar{x}_{2}=0$ in $[\overline{3}]$, and vice versa.

The order of both the direct and inverse transformations is $\sigma_{2,1,1}$ $+\sigma_{2,1,2}+3$, where $\sigma_{2,1,1}$ and $\sigma_{2,1,2}$ are any positive integers; one, but not both, of $\sigma_{2,1,1}$ and $\sigma_{2,1,2}$ may be zero.

$B^{(1)}$ is a $\left(\sigma_{2,1,1}+\sigma_{2,1,2}+1\right)$-fold $F$-line whose $P$-surface in $[\overline{3}]$ is of order $\sigma_{2,1,1}+\sigma_{2,1,2}+2$, having $\bar{x}_{1}=\bar{x}_{2}=0$ as $\left(\sigma_{2,1,1}+\sigma_{2,1,2}\right)$-fold line and (1000) as double point. $B^{(2)}$ is a simple $F$-curve of order $3\left(\sigma_{2,1,1}+\sigma_{2,1,2}+1\right)$, intersecting $B^{(1)}$ in $3\left(\sigma_{2,1,1}+\sigma_{2,1,2}\right)$ points. Its $P$-surface is ruled, consisting of the bisecants of curve $M$ that intersect line $\bar{x}_{1}=\bar{x}_{2}=0 . B_{2}^{(1)}=B_{1}^{(2)}=0$ is a simple $F$-conic. It intersects line $B^{(1)}$ in two points and curve $B^{(2)}$ in three points not on $B^{(1)}$. Its $P$-surface is the plane $\bar{x}_{2}=0$.

In $[\overline{3}], \bar{x}_{1}=\bar{x}_{2}=0$ is a $\left(\sigma_{2,1,1}+\sigma_{2,1,2}+1\right)$-fold $F$-line. Its $P$-surface is $B_{1}^{(2)}=0$, which contains $B^{(1)}$ as $\left(\sigma_{2,1,1}+\sigma_{2,1,2}\right)$-fold line and $B^{(2)}$ simply. Point (1000) is an isolated double $F$-point whose image in [3] is the plane $B_{2}^{(1)}=0$, containing line $B^{(1)}$ and the $F$-conic. $M$ is a simple $F$-curve whose order is $3 \sigma_{2,1,1}+3 \sigma_{2,1,2}+5$ and on which (1000) is a triple point. Its $P$-surface is ruled, consisting of the bisecants of $B^{(2)}$ that intersect $B^{(1)}$.

Finally there is the type

$$
\begin{array}{ccc}
1 & 1 & 1 \\
& \sigma_{2,1,1} & \sigma_{3,1,1} \\
& & \sigma_{3,1,2}
\end{array}
$$

Cremona transformation in $S_{3}$, where $\sigma_{2,1,1}$ and $\sigma_{3,1,2}$ are any positive integers while $\sigma_{3,1,1}$ is any positive integer or zero. $B^{(1)}$ is a line and $B^{(2)}$ is a space curve of order $2 \sigma_{2,1,1}+1$ having $2 \sigma_{2,1,1}$ points on $B^{(1)}$. This transformation sends the rays of the line congruence in [3], consisting of the lines that intersect both $B^{(1)}$ and $B^{(2)}$, over into the rays of the star on (0001) in [3] , and vice versa. It is a monoidal transformation since the homaloids in $[\overline{3}]$ are monoids.

The orders of the direct and inverse transformations are $\sigma_{3,2,1} \sigma_{2,1,1}$ $+\sigma_{3,2,1}+\sigma_{3,1,1}+\sigma_{2,1,1}+3$ and $\sigma_{3,2,1}+\sigma_{3,1,1}+\sigma_{2,1,1}+3$, respectively.

$B^{(1)}$ is a $\left(\sigma_{3,2,1} \sigma_{2,1,1}+\sigma_{3,1,1}+\sigma_{2,1,1}+1\right)$-fold $F$-line whose image in $[\overline{3}]$ is a surface of order $\sigma_{3,2,1}+\sigma_{3,1,1}+\sigma_{2,1,1}+2 . B^{(2)}$ is a $\left(\sigma_{3,2,1}+1\right)$ fold $F$-curve whose order is $2 \sigma_{2,1,1}+1$ and which intersects $B^{(1)}$ in $2 \sigma_{2,1,1}$ points. Its $P$-surface is of order $\sigma_{3,2,1}+\sigma_{3,1,1}+2 \sigma_{2,1,1}+2 . B^{(3)}$ is a simple $F$-curve of order $2 \sigma_{3,2,1} \sigma_{3,1,1}+2 \sigma_{3,2,1} \sigma_{2,1,1}+2 \sigma_{3,2,1}+2 \sigma_{3,1,1}+1$. Its $P$-surface is a cone of order $2 \sigma_{3,2,1}+2 \sigma_{3,1,1}+\sigma_{2,1,1}+2$ having (0001) as vertex and $M$ as base curve, and on which $\bar{x}_{2}=\bar{x}_{3}=0$ is $\left(\sigma_{3,2,1}+2\right)$ fold line. $B_{2}^{(1)}=B_{1}^{(2)}=0$ is a simple $F$-line intersecting $B^{(1)}, B^{(2)}$, and 
$B^{(3)}$. Its $P$-surface is the plane $\bar{x}_{2}=0 . B_{2}^{(1)}=B_{1}^{(3)}=0$ is a simple plane $F$-curve of second species whose order is $\sigma_{3,2,1}+1$ and which intersects $B^{(1)}$ in $\sigma_{3,2,1}+1$ points and $B_{2}^{(1)}=B_{1}^{(2)}=0$ in $\sigma_{3,2,1}+1$ points. Its image in $[\overline{3}]$ is the $F$-line $\bar{x}_{2}=\bar{x}_{3}=0 . B_{2}^{(2)}=B_{1}^{(3)}=0$ is a simple $F$-curve of order $\sigma_{3,1,1}+\sigma_{2,1,1}+1$ whose image is the plane $\bar{x}_{3}=0$.

As to the $F$-system in $[\overline{3}],(0001)$ is a $\left(\sigma_{3,2,1}+\sigma_{3,1,1}+\sigma_{2,1,1}+2\right)$-fold isolated $F$-point whose $P$-surface in [3] is $B_{1}^{(3)}=0$. Point (1000) is an isolated $\left(\sigma_{3,2,1}+2\right)$-fold $F$-point whose image is the plane $B_{2}^{(1)}=0$. $\bar{x}_{1}=\bar{x}_{2}=0$ is a $\left(\sigma_{3,1,1}+\sigma_{2,1,1}+1\right)$-fold $F$-line whose $P$-surface is $B_{1}^{(2)}=0$, of order $\sigma_{2,1,1}+1$, on which $B^{(1)}$ is a $\sigma_{2,1,1}$-fold line and $B^{(2)}$ is a simple curve. $\bar{x}_{2}=\bar{x}_{3}=0$ is a $\left(\sigma_{3,2,1}+1\right)$-fold $F$-line of second species whose image is the $F$-curve $B_{2}^{(1)}=B_{1}^{(3)}=0$ in [3]. $\bar{x}_{3}=\bar{x}_{4}=0$ is a simple $F$-line and its $P$-surface is $B_{2}^{(2)}=0 . M$ is a simple $F$-curve of order $2 \sigma_{3,2,1} \sigma_{3,1,1}$ $+\sigma_{3,2,1} \sigma_{2,1,1}+3 \sigma_{3,2,1}+3 \sigma_{3,1,1}+3 \sigma_{2,1,1}+3$. Its image is the ruled surface whose generators intersect $B^{(1)}, B^{(2)}$, and $B^{(3)}$, each once. To each point of $M$ corresponds a generator of the ruled surface.

It is worth noting that the coefficients in this transformation can be specialized so that the linear congruence in [3] degenerates into a $(k, 1)$ correspondence between the planes on line $B^{(1)}$ and the points of $B^{(1)}$. A generic point $P$ of [3] determines with $B^{(1)}$ a plane and this plane in turn determines one point 0 on $B^{(1)}$. Line $P O$ is the unique ray of the congruence through $P .^{7}$

Again, the coefficients of the general transformation can be specialized so that $B^{(2)}$ becomes a plane curve of order $k$ with a $(k-1)$-fold point.

\section{UnIVERSITY OF ARIZONA}

7 Purcell, loc. cit., p. 68. 